\title{
Bacteroides fragilis Prevents Clostridium difficile Infection in a Mouse Model by Restoring Gut Barrier and Microbiome Regulation
}

OPEN ACCESS

Edited by: George Grant, University of Aberdeen, United Kingdom

Reviewed by:

Nobuhiko Kamada, University of Michigan Health System,

United States

Shailesh K. Shahi, The University of lowa, United States

Reetta Satokari,

University of Helsinki, Finland

*Correspondence: Hongying Fan

biofhy@126.com

Xinmei Zhao zxm03172011498@163.com

Fachao Zhi

zhifc41532@163.com

Specialty section: This article was submitted to

Infectious Diseases,

a section of the journal

Frontiers in Microbiology

Received: 09 September 2018 Accepted: 19 November 2018 Published: 21 December 2018

Citation:

Deng $H$, Yang S, Zhang Y, Qian $K$, Zhang Z, Liu Y, Wang Y, Bai Y, Fan H, Zhao $X$ and Zhi F (2018) Bacteroides

fragilis Prevents Clostridium difficile Infection in a Mouse Model by Restoring Gut Barrier and Microbiome Regulation. Front. Microbiol. 9:2976. doi: 10.3389/fmicb.2018.02976
Huimin Deng ${ }^{1}$, Siqi Yang ${ }^{1}$, Yucheng Zhang ${ }^{1}, K_{\text {ai Qian }}{ }^{1}$, Zhaohui Zhang ${ }^{1}$, Yangyang Liu ${ }^{2}$, Ye Wang ${ }^{2}$, Yang Bai ${ }^{1}$, Hongying Fan ${ }^{3 *}$, Xinmei Zhao ${ }^{1 *}$ and Fachao Zhi ${ }^{1 *}$

\footnotetext{
' Guangdong Provincial Key Laboratory of Gastroenterology, Department of Gastroenterology, Institute of Gastroenterology of Guangdong Province, Nanfang Hospital, Southern Medical University, Guangzhou, China, ${ }^{2}$ Guangzhou ZhiYi Biotechnology Co., Ltd., Guangzhou, China, ${ }^{3}$ Guangdong Provincial Key Laboratory of Tropical Disease Research, School of Public Health, Southern Medical University, Guangzhou, China
}

Clostridium difficile is currently the leading cause of nosocomial infection. Antibiotics remain the first-line therapy for $C$. difficile-associated diseases (CDAD), despite the risks of resistance promotion and further gut microbiota perturbation. Notably, the abundance of Bacteroides fragilis was reported to be significantly decreased in CDAD patients. This study aimed to clarify the prophylactic effects of $B$. fragilis strain ZY-312 in a mouse model of $C$. difficile infection (CDI). The CDI mouse model was successfully created using C. difficile strain VPI 10463 spores, as confirmed by lethal diarrhea (12.5\% survival rate), serious gut barrier disruption, and microbiota disruption. CDI model mice prophylactically treated with $B$. fragilis exhibited significantly higher survival rates (100\% in low dosage group, $87.5 \%$ in high dosage group) and improved clinical manifestations. Histopathological analysis of colon and cecum tissue samples revealed an intact gut barrier with strong ZO-1 and Muc-2 expression. The bacterial diversity and relative abundance of gut microbiota were significantly improved. Interestingly, the relative abundance of Akkermansia muciniphila was positively correlated with $B$. fragilis treatment. In vitro experiments showed that $B$. fragilis inhibited $C$. difficile adherence, and attenuated the decrease in CDI-induced transepithelial electrical resistance, ZO1 and MUC-2 loss, and apoptosis, suggesting that $B$. fragilis protected against CDI possibly by resisting pathogen colonization and improving gut barrier integrity and functions. In summary, $B$. fragilis exerted protective effects on a CDI mouse model by modulating gut microbiota and alleviating barrier destruction, thereby relieving epithelial stress and pathogenic colitis triggered by $C$. difficile. This study provides an alternative preventative measure for $\mathrm{CDI}$ and lays the foundations for further investigations of the relationships among opportunistic pathogens, commensal microbiota, and the gut barrier.

Keywords: next-generation probiotic, gut barrier, gut microbiota, Clostridium difficile, commensal bacteria 


\section{INTRODUCTION}

The overuse of antibiotics is currently regarded as the most common reason for disturbance of gut microbiota, and if the disruption reaches a certain level, the host can develop Clostridium difficile-associated disease (CDAD) (He et al., 2013; Tang et al., 2016). C. difficile, the leading cause of AAD, is a Gram-positive, spore-forming, opportunistic pathogenic anaerobe. As the major culprit for nosocomial infections, it has captured wide public attention for its high morbidity and mortality rates (Kelly and LaMont, 2008; He et al., 2013; Lessa et al., 2015). CDAD is characterized by a spectrum of diseases ranging from clinical diarrhea to pseudomembranous colitis and toxic megacolon. The in vivo pathogenesis of $C$. difficile is mainly dependent on two macromolecular toxins, toxin A and toxin B. These toxins target and destroy intestinal epithelial cells by modifying cytoskeletal components and disrupting tight cellular junctions, ultimately leading to apoptosis and disruption of gut barrier integrity (Pothoulakis, 2000; Rineh et al., 2014).

The first-line therapy for C. difficile infection (CDI) remains antibiotics, mainly MTZ, vancomycin, and fidaxomicin (Mushtaq, 2018). Although antibiotics are regarded as the most efficient therapy in clinical practice, the risks of resistance promotion and further gut microbiota perturbation cannot be ignored. Therefore, effective alternative non-antibiotic therapies are urgently needed and have been researched for several years. Recently, bacteriotherapy has gradually become accepted as a solution for CDI treatment (Lawley et al., 2012; van Nood et al., 2013; Shen et al., 2017; Mills et al., 2018). An integrated gut barrier and a healthy microbiota net are vital to resist $C$. difficile. Therefore, complementing bacteria in a timely manner to rebuild a health-associated microbiota net seems more reasonable. Recent clinical studies have proven that FMT has excellent therapeutic effects in CDI patients (van Nood et al., 2013; Kelly and Tebas, 2018). However, despite the high clinical remission rate, FMT therapy still has safety concerns including the risk of unexpected infections by undetected pathogenic bacteria. Moreover, the exact mechanisms of FMT therapy for CDI remission remain to be clearly elucidated.

Bacteroides fragilis (B. fragilis) was reported to have an inverse association with $C$. difficile (Goldberg et al., 2014) in a study of 59 patients including CDI or non-CDI patients, indicating that $B$. fragilis probably plays a role in the protection from CDI. We previously demonstrated that $B$. fragilis exerted protective effects in an $\mathrm{AAD}$ rat model through microbiome regulation and

\footnotetext{
Abbreviations: AAD, antibiotic-associated diarrhea; BFT, Bacteroides fragilis treatment; BHI, brain heart infusion; BSA, bovine serum albumin; CDAD, Clostridium difficile associated disease; CDI, Clostridium difficile infection; DAPI, 2-(4-Amidinophenyl)-6-indolecarbamidine; DNA, deoxyribonucleic acid; FBS, fetal bovine serum; FITC, fluoresceine isothiocyanate; FMT, fecal microbiota transplantation; MTZ, metronidazole; NMDS, non-metric multidimensional scaling; OTU, operational taxonomic unit; PAS, Periodic acid-Schiff; PBS, phosphate-buffered saline; PBST, phosphate-buffered saline containing $0.1 \%$ Tween-20; PCR, polymerase chain reaction; PI, propidium iodide; RPMI, roswell park memorial institute; rRNA, ribosomal ribonucleic acid; SEM, standard error of mean; TEER, transepithelial electrical resistance; TSB, tryptone soy broth; ZO-1, zona occludens-1.
}

gut barrier integrity restoration (Zhang et al., 2018). Therefore, in the present study, we examined the protective effects of B. fragilis in a mouse model of CDI and explored the possible mechanism involving microbe-epithelium interactions. As we know, an intact gut barrier is vital for natural host defenses against $C$. difficile (Buonomo et al., 2016; Winston and Theriot, 2016; Crobach et al., 2018). Many factors contribute to intestinal barrier function, including epithelial integrity, a thick mucus layer, healthy gut microbiota, and a stable immune system (Bron et al., 2017). Normally, colon epithelial cells are sealed with tight junction proteins to form a strong barrier, while mucins act as an impermeable diffusion barrier that lies over the epithelium. Muc-2 protein is the most common type of mucin expressed in the colon and $\mathrm{ZO}-1$ protein is a representative tight junction protein. In this study, we explored the influence of $B$. fragilis on Muc-2 and ZO-1 expression in vivo and in vitro.

Our data demonstrated that $B$. fragilis ZY-312 significantly reduced mortality in the CDI mouse model, associated with the restoration of gut barrier integrity and regulation of microbiota. In vitro experiments suggested that $B$. fragilis inhibited $C$. difficile adherence to colon cells and reduced $C$. difficile-induced apoptosis, and Muc-2 and ZO-1 loss.

\section{MATERIALS AND METHODS}

\section{Ethics Statement}

All animal experiments were approved by Nanfang Hospital Animal Ethics Committee (approval number: NFYY-2014-123) and were in accordance with relevant ethical principles and guidelines set by the Animal Welfare Act and the NIH Guide for the Care and Use of Laboratory Animals. Experiments involving isolation of B. fragilis strain ZY-312 from infant fecal samples were approved by the Medical Ethics Committee of Nanfang Hospital (approval number: NFEC-2014-040).

\section{Bacteria Strains, Cell Lines, and Culture Conditions}

Bacteroides fragilis strain ZY-312 was described in detail in our previous report (Deng et al., 2016; Wang et al., 2017), and the same cultivation conditions were used. C. difficile strain VPI 10463 was purchased from the American Type Culture Collection (Manassas, VA, United States) and verified by PCR amplification. Strain VPI 10463 was cultured anaerobically in sterile tubes containing $4 \mathrm{~mL}$ of TSB supplemented with $20 \% \mathrm{FBS}$ at $37^{\circ} \mathrm{C}$ in an anaerobic glove box (DG250; Don Whitley Scientific, Bingley, United Kingdom) for $24 \mathrm{~h}$.

The human colon carcinoma cell lines HT-29, Caco-2, IEC6, and Vero were provided by Southern Medical University (Guangzhou, China), and routinely cultured in RPMI 1640 medium (Gibco, Life Technologies, Carlsbad, CA, United States) supplemented with $10 \%$ heat-inactivated FBS (PAN, Aidenbach, Germany), penicillin (100 U/ml), and streptomycin (100 ng/ml) in an incubator at $37^{\circ} \mathrm{C}$ under $95 \%(\mathrm{v} / \mathrm{v})$ humidified air and $5 \%$ (v/v) $\mathrm{CO}_{2}$. 


\section{Preparation of C. difficile VPI 10463 Spores}

Sporulation of VPI 10463 was induced as described previously (Sorg and Sonenshein, 2008). Briefly, strain VPI 10463 was stored at a ratio of $1: 1$ with glycerin at $-80^{\circ} \mathrm{C}$ before sporulation. For sporulation, $20 \mu \mathrm{L}$ of the stored solution was seeded in $\mathrm{BHI}$ agar (BD Biosciences, San Jose, CA, United States) containing $0.1 \%$ taurocholate (Sigma, St. Louis, MO, United States) and incubated anaerobically at $37^{\circ} \mathrm{C}$ for 7 days. More than $80 \%$ of spores in individual microscope fields were considered eligible for subsequent experiments (Supplementary Figure S1A). After incubation, $3 \mathrm{~mL}$ of sterilized ice-cold water per agar plate was used to wash out the vegetative cell-spore mixed colonies. The resulting solution was collected in a sterilized tube, centrifuged at $3000 \mathrm{rpm}$ for $5 \mathrm{~min}$, and washed once. The pellet was resuspended in $10 \mathrm{~mL}$ of $50 \%(\mathrm{w} / \mathrm{v})$ sucrose solution and centrifuged at $3000 \mathrm{rpm}$ for $20 \mathrm{~min}$. After five washes with cold water by centrifugation at $3000 \mathrm{rpm}$ for $20 \mathrm{~min}$, the samples were heated to $60^{\circ} \mathrm{C}$ for $30 \mathrm{~min}$ to kill the vegetative bacteria. The concentration of the spore solution was determined by a serial dilution method on BHI agar containing $0.1 \%$ taurocholate. The spore solution was stored at $-80^{\circ} \mathrm{C}$ until use. The toxicity of the spores was determined using a C. Diff Quik Chek Complete Detection Kit (Techlab, Blacksburg, VA, United States).

\section{Animals and CDI Model}

To induce the CDI model, 6-8-week-old male C57BL/6 mice were obtained from the Animal Experiment Center of Guangdong Province (China) and reared under specific pathogen-free conditions in the Southern Medical University animal facility. All animals had appropriate space, with free access to tap water and a standard rodent diet. The CDI mouse model was induced as previously described (Hutton et al., 2014; Yan et al., 2014) with some modifications. The detailed procedure is shown in Figure 1A. Water containing antibiotics, comprising of kanamycin $(0.8 \mathrm{mg} / \mathrm{mL}$; Sigma $)$, gentamicin (0.07 mg/mL; Sigma), colistin (0.1135 mg/mL; Sigma), MTZ (0.43 mg/mL; Sigma) and vancomycin (0.09 mg/mL; Sigma), was provided for 7 consecutive days starting on Day -9 , and then replaced with autoclaved water from Day -2 . A dose of clindamycin (10 mg/kg; Sigma) was given intraperitoneally at Day -1 . The prepared toxigenic spores were administered to the model mice at a dose of $3 \times 10^{8}$ colony forming units (cfu) by oral gavage from Day 0 for 3 consecutive days. The animals were observed every $4 \mathrm{~h}$, mainly for the presence of diarrhea and death after the spore challenge. Body weight was measured daily from Day -9 to Day 2 (data after Day 3 not provided due to death of the mice). Cecal contents and cecum and colon tissue samples were collected, and $C$. difficile toxin was detected in supernatants of cecal contents, soon after the death of mice. The surviving mice were euthanized by $\mathrm{CO}_{2}$ inhalation at Day 7 . Tissue samples including cecum and colon tissues were collected for histopathologic and immunohistochemical analysis and cecal contents were collected for $16 \mathrm{~S}$ rRNA analysis. Mean optical density of the immunohistochemical images were qualified using Image J 1.51 software.

A

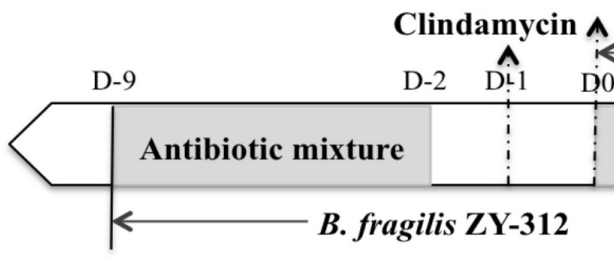

B

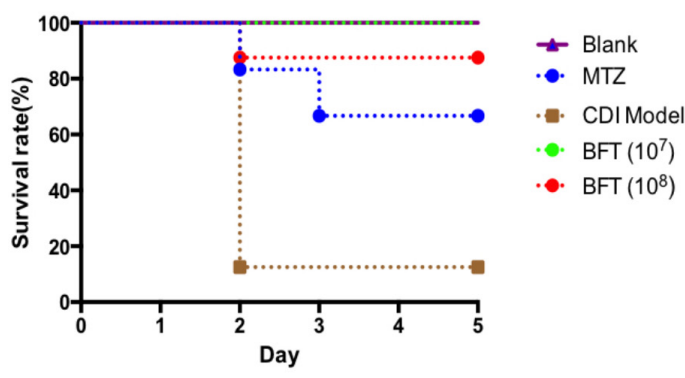

Metronidazole $\longrightarrow \hat{i}$

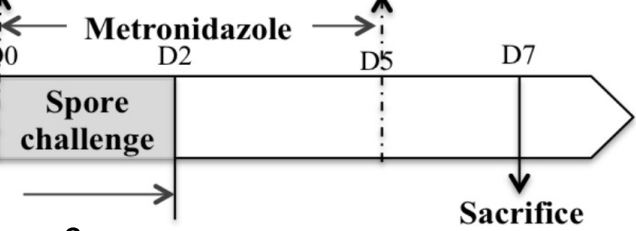

C

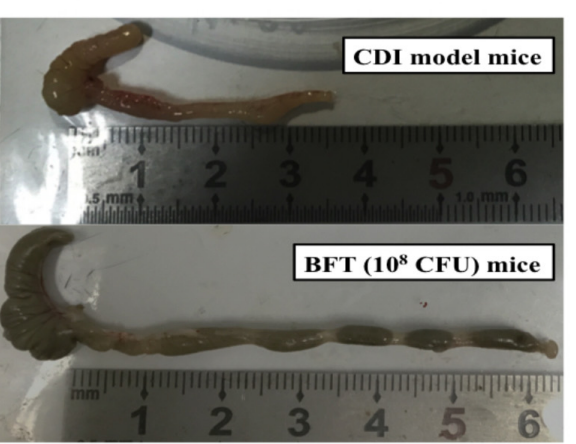

FIGURE 1 | Bacteroides fragilis ZY-312 prevents CDI-associated death. (A) Experimental design for B. fragilis ZY-312 prophylactic treatment of the CDI model mice. The first step of inducing the $C D I$ mouse model was to use a large dosage of antibiotics to disrupt the normal gut microbiota. From $D-9$ to $D-2$, drinking water with antibiotics including kanamycin $(0.8 \mathrm{mg} / \mathrm{mL})$, gentamicin $(0.07 \mathrm{mg} / \mathrm{mL})$, colistin $(0.1135 \mathrm{mg} / \mathrm{mL})$, metronidazole $(0.43 \mathrm{mg} / \mathrm{mL})$, and vancomycin $(0.09 \mathrm{mg} / \mathrm{mL})$ was offered to male C57BL/6 mice (6-8 weeks old, $n=6-8)$. Clindamycin $(10 \mathrm{mg} / \mathrm{kg})$ was intraperitoneally injected the day $(\mathrm{D}-1)$ after the cessation of drinking water with antibiotics. Then the mice were orally challenge with $3 \times 10^{8} \mathrm{cfu}$ of Clostridium difficile VPI 10463 spores from D0 to D2. To explore the prophylactic effects of B. fragilis on preventing C. difficile-associated diseases, $1 \times 10^{7}$ or $1 \times 10^{8} \mathrm{cfu} /$ day $B$. fragilis were given, respectively, to CDI model mice from D -9 to D2. Metronidazole (1 mg/day) was given orally from D0 to D5, as the positive control. (B) Survival rates of mice in the Blank, MTZ (Metronidazole treated), CDI model, and $\mathrm{BFT}$ (B. fragilis treatment) groups. (C) Visual observations of colon tissues from CDI model mice (top) and BFT (10 ${ }^{8}$ cfu) mice (means \pm SEM; $n=6-8$ per group). 


\section{Grouping}

Mice were randomly assigned to seven groups $(n=6-8$ mice/group), including Blank, CDI (C. difficile infection) model, BFT (B. fragilis prophylactic treatment $\left.10^{7} \mathrm{cfu}\right)$, BFT $\left(10^{8}\right.$ cfu), MTZ (metronidazole), BFC (B. fragilis control) and Saline groups. Mice in the Blank group were reared from beginning to end without any treatment. Mice in the CDI model group were treated as described in Section "Animals and CDI Model." The BFT groups were used to explore the prophylactic effects of $B$. fragilis on preventing CDAD, and $1 \times 10^{7}$ or $1 \times 10^{8}$ cfu/day $B$. fragilis were given, respectively, to CDI model mice from D -9 to D2. Mice in the MTZ group were given MTZ ( $1 \mathrm{mg} /$ day) orally from D0 to D5, as the positive control. Mice in the Saline group drank antibiotic water from $D-9$ to $D-2$ and were injected with clindamycin at $\mathrm{D}-1$, to observe the effect of antibiotics alone on mouse gut microbiota. Mice in the BFC control group drank antibiotic water from D -9 to $D$ -2 and were injected with clindamycin at $\mathrm{D}-1$ and received $1 \times 10^{8} \mathrm{cfu} /$ day $B$. fragilis from the onset $(\mathrm{D}-9)$ to $\mathrm{D} 2$, to observe the effect of $B$. fragilis alone on mice with disturbed gut microbiota.

\section{Microbial DNA Extraction From Cecal Content and 16S rRNA Gene Sequencing}

Microbial DNA was extracted from the mouse cecal content samples using a QIAamp Stool DNA Mini Kit (Qiagen, Hilden, Germany) following the manufacturer's instructions. The V4 region of the 16S rRNA gene was amplified from the cecal DNA samples using the primer pair 515F/806R. All libraries were sequenced using the Ion $\mathrm{S}^{\mathrm{TM}}$ XL platform (Thermo Fisher, Waltham, MA, United States) by Nuohezhiyuan Co. (China).

Sequencing analysis was performed in the Quantitative Insights into Microbial Ecology framework (QIIME, Version 1.9.1) as described previously (Kuczynski et al., 2012). Briefly, low-quality bases were initially screened using Cutadapt (Version 1.9.1), and then pair reads were filtered using the UCHIME Algorithm. After filtering, we obtained a total of 2,412,968 raw reads from 28 cecal content samples. The average number of raw reads per sample was 86177 ( $\pm 6690 \mathrm{SD})$, and the average number of clean reads per sample was 81207 ( \pm 6025 SD). Clean reads were clustered as OTUs based on the threshold of $97 \%$ identity, and representative sequences were annotated (top 10 were listed). The Chao diversity index and the number of observed species per sample were used as $\alpha$-diversity metrics. $\beta$-diversity was calculated using unweighted UniFrac distances and represented in Non-Metric Multi-Dimensional Scaling (NMDS) analyses. The $T$-test method was used to analyze differences in species richness between groups.

Raw data for all samples used in this study have been deposited in the BioProject database at NCBI available as BioProject ID PRJNA505127, at http://www.ncbi.nlm.nih.gov/bioproject/ 505127.

\section{Histological Analysis}

Cecum and colon tissue samples were fixed in $4 \%$ paraformaldehyde, embedded in paraffin, and cut into $4 \mu \mathrm{m}$ sections. The sections were stained with hematoxylin-eosin and observed under an optical microscope (TE2000-U; Nikon, Tokyo, Japan). For immunohistochemical staining, the paraffinembedded sections were deparaffinized, rehydrated, subjected to heat-induced antigen retrieval in citrate buffer, and blocked with PBS containing $1 \%(\mathrm{w} / \mathrm{v})$ BSA. The sections were then incubated with anti-MUC-2, anti-ZO-1, anti-Occludin primary antibodies (Wuhan Service Biotechnology Co., Ltd., China) at a dilution of 1:100, followed by a biotinylated secondary antibody (Wuhan Service Biotechnology Co., Ltd.). Bound antibodies were visualized using $3,3^{\prime}$-diaminobenzidine as a substrate.

\section{Western Blotting}

Mouse colon tissues were lysed using radio immunoprecipitation assay lysis buffer (Beyotime, Haimen, China), and tissue lysates were centrifuged at $14,000 \times g$ for $30 \mathrm{~min}$. Supernatants were collected and mixed with $5 \times$ sodium dodecyl sulfate (SDS) sample buffer. The samples were separated by SDSpolyacrylamide gel electrophoresis using $12 \%$ acrylamide gels, and then transferred to polyvinylidene fluoride membranes (Millipore, Billerica, MA, United States). Following incubation with primary and secondary antibodies, protein bands were detected with Clarity ${ }^{\mathrm{TM}}$ Western ECL Substrate (Bio-Rad, United States) and analyzed using the Biolmaging System (UVP, CA, United States). The following antibodies were used: rabbit anti-caspase 3, (Cat.: AF6311, Affinity, United States), rabbit anti-bcl-2(Cat.: AF6139, Affinity, United States), rabbit anti-bax (Cat.: AF0120, Affinity, United States), mouse anti- $\beta$-actin (Cat.: AC001-M, Dingguo, China).

\section{Adhesion Assays}

HT-29 cells were seeded in 24-well plates at $1 \times 10^{5}$ cells/well and incubated overnight. $B$. fragilis ZY-312 cells were harvested and adjusted to $5 \times 10^{8} \mathrm{cfu} / \mathrm{mL}$, and $C$. difficile cells were adjusted to $5 \times 10^{9} \mathrm{cfu} / \mathrm{mL}$. Bacterial cells in a volume of $20 \mu \mathrm{L}$ were added to relevant wells in the following groups: A: positive control group, cells infected with $C$. difficile only; B: competition group, cells co-infected with $B$. fragilis and $C$. difficile; $C$ : exclusion group, B. fragilis cells added for an hour prior to co-culture with $C$. difficile; D: substitution group, $C$. difficile cells added for an hour and prior to co-culture with B. fragilis. All cells were incubated anaerobically at $37^{\circ} \mathrm{C}$ for $2 \mathrm{~h}$ in total. After the incubation, non-adherent bacteria were removed by three washes with PBS and lysed with $1 \mathrm{~mL}$ of distilled water. The adherent C. difficile cells were serially diluted and spread on TSB plates containing $1 \mu \mathrm{g} / \mathrm{mL}$ clindamycin. The relative adhesion rates were calculated as follows: relative adhesion rate $=$ adhesion rate/positive control adhesion rate $\times 100 \%$.

\section{Transepithelial Electrical Resistance (TEER) Measurement}

To determine the protective effect of $B$. fragilis ZY-312 on the permeability of colon cell monolayers, $2 \times 10^{4}$ Caco- 2 cells were seeded on 24-well transwell filters and cultured in medium containing 20\% FBS for 21 days to allow polarization. The culture medium was replaced every other day. At Day 21, the cells 
had grown to $3 \times 10^{4}$ cells in all wells and were washed three times with PBS. Next, $400 \mu \mathrm{L}$ and $1 \mathrm{~mL}$ of culture medium was added into the upper and lower compartments, respectively. Six groups were created as follows: control group, cells cultured without any treatment; ZY-312 group, cells incubated with $3 \times 10^{7}$ cfu B. fragilis ZY-312; C. difficile group, cells incubated with $3 \times 10^{6} \mathrm{cfu} C$. difficile VPI 10463; competition group, exclusion group, and substitution group, as per the adhesion assays (see section "Western Blotting"). The TEER was measured with an EVOM2 (World Precision Instruments, Sarasota, FL, United States) in each transwell and TEER determined by the following equation: $\mathrm{TEER}=($ target value - initial value $) \times 0.33$.

\section{Immunofluorescence Assays for Zona Occludens-1 (ZO-1) and Muc-2}

The expression level of the tight junction protein $\mathrm{ZO}-1$ in Caco2 cells and the mucin Muc- 2 in HT-29 cells was determined by immunofluorescence. The groups were the same as those for the TEER assays (see section "Adhesion Assays"). Before observation, $4 \times 10^{4}$ Caco- 2 or HT-29 cells were seeded in $10-\mathrm{mm}$ confocal dishes, allowed to adhere, and washed with PBS, before addition of bacterial cells as described above. The colon cells were infected with $4 \times 10^{7} \mathrm{cfu} B$. fragilis and $4 \times 10^{6} \mathrm{cfu} C$. difficile in each group. After the incubation, the cells were washed twice with PBS containing $0.1 \%$ Tween20 (PBST), fixed with $200 \mu \mathrm{L}$ of $2 \%$ paraformaldehyde for $30 \mathrm{~min}$, washed three times with cold PBST, and blocked for $2 \mathrm{~h}$. The blocking solution was composed of PBST containing $10 \%$ goat serum, 1\% BSA and 3\% glycine. Next, the cells were incubated with anti-ZO-1 or anti-Muc-2 antibodies (Abcam, Cambridge, United Kingdom) in PBST containing 1\% BSA at $4^{\circ} \mathrm{C}$ overnight, washed three times with PBST, incubated with a secondary antibody conjugated with DyLight 488 (Abbkine, Wuhan, China) at room temperature for $1 \mathrm{~h}$, washed three times with PBST, and incubated with $10 \mu \mathrm{g} / \mathrm{ml}$ DAPI for $10 \mathrm{~min}$. After removal of the DAPI solution, one drop of fluorescence quenching agent was added to the cell surface and the cells were examined under a fluorescence microscope (TE2000-U; Nikon).

\section{Periodic Acid-Schiff (PAS) Staining for Muc-2 Protein}

Periodic acid-Schiff staining reagent (SenBeiJia, Nanjing, China) was used to visualize Muc-2 in HT-29 cell monolayers according to the manufacturer's protocol. Briefly, $1 \times 10^{5}$ cells were seeded in 24-well plates and infected with $1 \times 10^{7} \mathrm{cfu}$ $C$. difficile and $1 \times 10^{8} \mathrm{cfu} B$. fragilis for $2 \mathrm{~h}$. The groups and treatments were as described in Section "Western Blotting." After the treatment, the cells were washed three times with PBS, incubated with $300 \mu \mathrm{L}$ of PAS solution for $15 \mathrm{~min}$ at room temperature, washed, oxidized with $200 \mu \mathrm{L} /$ well periodic acid solution for $20 \mathrm{~min}$, washed, incubated with $200 \mu \mathrm{L} /$ well Schiff reagent for $30 \mathrm{~min}$, and washed twice with sodium sulfite solution. The cells were counterstained with hematoxylin and observed under an optical microscope (IMT-2, Olympus, Tokyo, Japan).

\section{Cell Apoptosis Assay}

A flow cytometry method was used to examine the effects of B. fragilis ZY-312 on C. difficile-induced apoptosis of HT29 cells. Briefly, $5 \times 10^{5}$ cells were inoculated into 35$\mathrm{mm}$ culture dishes and treated for $2 \mathrm{~h}$ in different groups as described in Section "Western Blotting" (multiplicity of infection: 1000 for B. fragilis and 100 for C. difficile). All cells were harvested by centrifugation, stained with Annexin V-FITC and PI, and analyzed by flow cytometry (FACSCalibur; BD Biosciences). Staining was also performed on Vero and IEC-6 cells to observe the protective effects of $B$. fragilis ZY312. These cells were treated as described above, stained with Annexin V-FITC and PI, and observed under a fluorescence microscope. Unstained cells were observed under an optical microscope.

\section{Statistical Analysis}

Data are presented as the mean \pm SEM, unless otherwise indicated. All cell experiments were performed three times independently and each experiment was performed in triplicate. Statistical analyses for the significance of differences in data were performed by a two-tailed $t$-test, repeated-measures analysis of variance, or one-way analysis of variance where appropriate. Values of $p<0.05$ were considered statistically significant.

\section{RESULTS}

\section{C. difficile VPI 10463 Spores Successfully Induced CDAD, Gut Microbiota Disorder, Gut Barrier Disruption, and Death}

In this study we successfully induced a CDI model by challenging antibiotic-treated mice with $C$. difficile spores. All CDI model mice (100\%) developed serious diarrheic symptoms (Table 1) and weight loss (Supplementary Figure S1C) soon after spore challenge, and $87.5 \%$ of the mice had died at Day 2 (Figure 1B). C. difficile toxin was detected in the cecal contents of all model mice (Supplementary Figure S1B). Significant histopathological damage was observed in the tissue samples (Figure 1C), especially in the gut epithelium (Figure 2). The colon structure was destroyed and enterocytes had died,

TABLE 1 | Incidence of Clostridium difficile-induced diarrhea and effect of treatment.

\begin{tabular}{lccc}
\hline Group & D1 & D2 & D3 \\
\hline Blank & 0 & 0 & 0 \\
CDI model & $87.5 \%(7 / 8)$ & $100 \%(8 / 8)$ & 0 \\
Metronidazole & 0 & $66.7 \%(4 / 6)$ & $20 \%(1 / 5)$ \\
Saline control & 0 & 0 & $33.3 \%(2 / 6)$ \\
BFC & 0 & $50 \%(3 / 6)$ & 0 \\
ZY-312 $\times 10^{7}$ & 0 & $37.5 \%(3 / 8)$ & 0 \\
ZY-312 $\times 10^{8}$ & $75 \%(6 / 8)$ & $37.5 \%(3 / 8)$ & $12.5 \%(1 / 8)$ \\
\hline
\end{tabular}

Clostridium difficile spores were used to challenge the antibiotic treated mice at D0, and D1, D2. D1, D2 and D3 means the first, second, and third day after D0, respectively. 


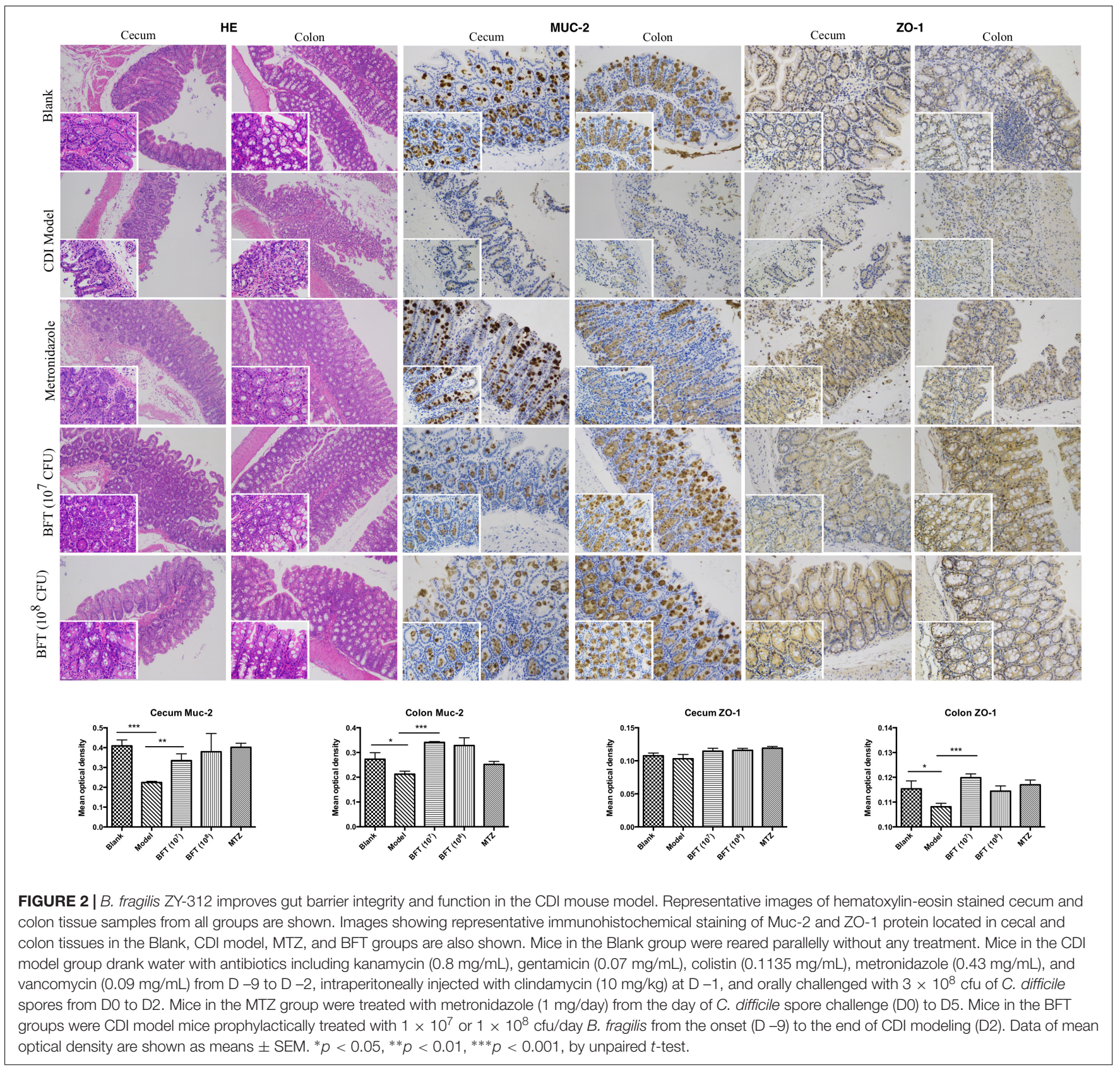

with obvious inflammatory cell infiltration, digestive gland destruction, and goblet cell loss, compared with the vehicle control group. Muc-2 and ZO-1 protein loss was confirmed by immunohistochemical analysis. In 16S rRNA analyses, the gut microbiota of antibiotic-treated mice was significantly perturbed after spore challenge (Supplementary Figures S4, S5). The OTU number (Figure 3C) was dramatically decreased and the relative abundance (Figure 3B) remained only two phyla, Proteobacteria and Firmicutes, while the richness of Bacteroidetes decreased to zero, consistent with a previous report (Wang et al., 2017). The cluster heat map further revealed that most of the altered phyla (Figure 3E) and species (Supplementary Figure S4) in the cecal contents belonged to the Firmicutes phylum.

\section{B. fragilis ZY-312 Reduces Diarrhea and Death, Likely by Regulating Gut Microbiota and Protecting the Gut Barrier}

In this study, we verified the protective effects of $B$. fragilis in a CDI mouse model. First, we tested the safety of a high dose $\left(1 \times 10^{8} \mathrm{cfu}\right)$ of $B$. fragilis in antibiotic-treated mice, no mice died in the BFC group and 50\% displayed mild diarrhea at Day 2, but soon recovered by Day 3 (Table 1 and Figure 2). Histopathological and immunohistochemical analyses provided consistent results (Supplementary Figure S2). Next, we prophylactically treated the CDI mice with $B$. fragilis $\left(1 \times 10^{7}\right.$ and 

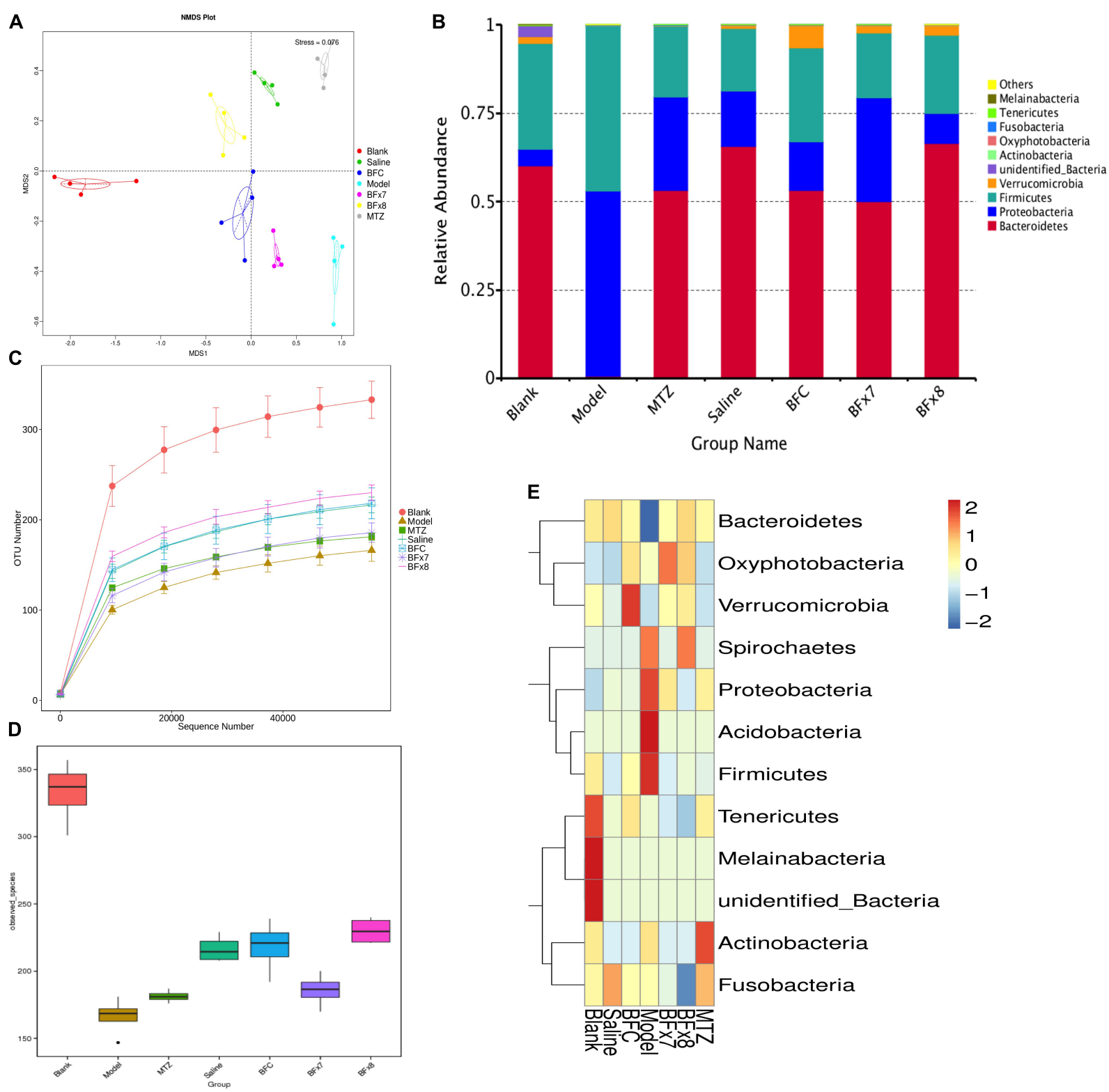

FIGURE 3 | Bacteroides fragilis ZY-312 regulates gut microbiota in the CDI mouse model. (A) Non-Metric Multi-Dimensional Scaling analyses based on OTUs in the Blank, Model, MTZ, Saline, BFC, BF $\times 7$, and BF $\times 8$ groups. (B) Relative abundance of the top 10 phyla in the gut microbiota of all groups. (C) Rarefaction curve showing the relative abundance of OTUs in all groups. (D) Observed species in all groups. (E) Cluster heat map indicating the richness of the top 12 phyla in all groups (Stress values $<0.2$ indicates significant differences between samples). Mice in the Blank group were reared parallelly without any treatment. Mice in the CDI model group drank water with antibiotics including kanamycin $(0.8 \mathrm{mg} / \mathrm{mL})$, gentamicin $(0.07 \mathrm{mg} / \mathrm{mL})$, colistin $(0.1135 \mathrm{mg} / \mathrm{mL}), \mathrm{metronidazole}(0.43 \mathrm{mg} / \mathrm{mL})$, and vancomycin $(0.09 \mathrm{mg} / \mathrm{mL})$ from $D-9$ to $D-2$, were intraperitoneally injected clindamycin $(10 \mathrm{mg} / \mathrm{kg})$ at $D-1$, orally challenged with $3 \times 10^{8} \mathrm{cfu}$ of $C$. difficile spores from D0 to D2. Mice in the MTZ group were treated with metronidazole (1 mg/day) from the day of spore challenge (D0) to D5. Mice in the BFT groups were CDI model mice prophylactically treated with $1 \times 10^{7} \mathrm{cfu} /$ day $\left(\mathrm{BF} \times 7\right.$ group) or $1 \times 10^{8} \mathrm{cfu} /$ day $(\mathrm{BF} \times 8$ group) $B$. fragilis from the onset $(\mathrm{D}-9)$ to the end of $\mathrm{CDI}$ modeling (D2). Mice in the Saline group drank antibiotic water from D -9 to D -2 and were injected with clindamycin at $D-1$, to observe the effect of antibiotics alone on mice gut microbiota. Mice in the BFC control group drank antibiotic water from $D-9$ to $D-2$, were injected with clindamycin at $D-1$ and received $1 \times 10^{8} \mathrm{cfu} /$ day $B$. fragilis from the onset $(\mathrm{D}-9)$ to $\mathrm{D} 2$, to observe the effect of $B$. fragilis alone on mice with antibiotic-induced dysbiosis.

$1 \times 10^{8} \mathrm{cfu} /$ day) and impressive protective effects were observed. All mice prophylactically treated with $B$. fragilis displayed much lower morbidity (Table 1) and mortality (Figure 1B) compared with the CDI model mice. The expressions of Muc-2 and ZO-1 proteins (Figure 2) were better preserved, and expressions of the apoptosis proteins (Supplementary Figure S2D) were inhibited in intestinal cells, indicating that $B$. fragilis can protect the gut epithelium of CDI model mice. Furthermore, 16S rRNA analyses revealed that $B$. fragilis was sufficient to regulate the cecal microbiota in the CDI model mice by increasing the 

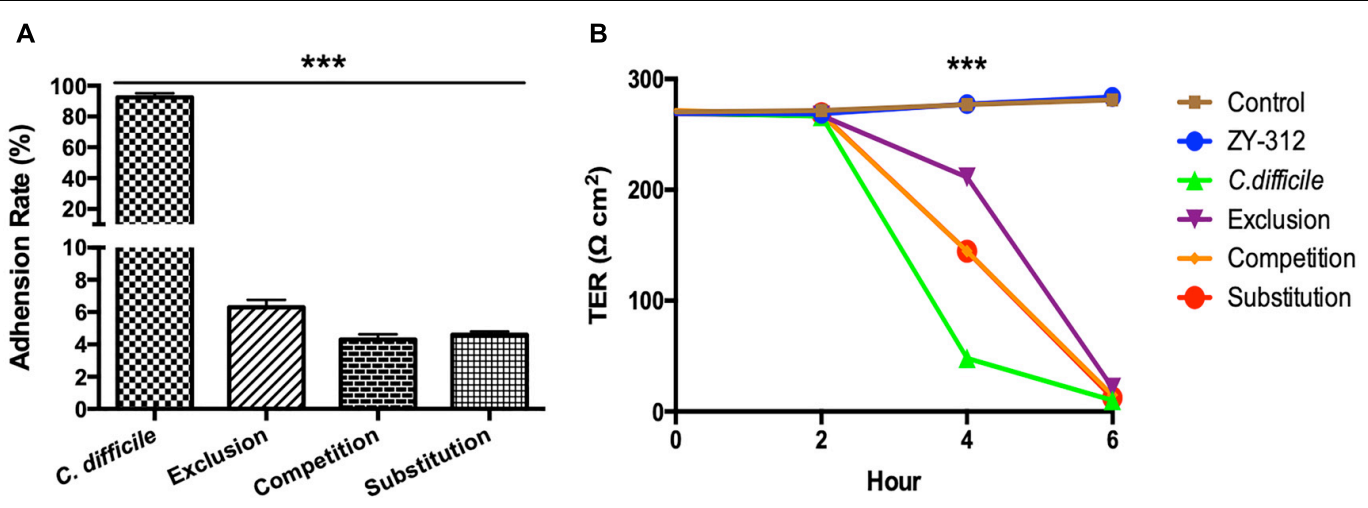

FIGURE 4 | Bacteroides fragilis ZY-312 inhibits C. difficile adherence and improves TEER. (A) Adherence to HT-29 cells in C. difficile, Exclusion, Competition and Substitution groups. C. difficile group, $1 \times 10^{5} \mathrm{HT}$-29 cells were infected with $1 \times 10^{7} \mathrm{cfu}$ C. difficile; Competition group, $1 \times 10^{5} \mathrm{HT}-29 \mathrm{cell}$ were co-infected with $1 \times 10^{8} \mathrm{cfu} B$. fragilis and $1 \times 10^{7} \mathrm{cfu} C$. difficile; Exclusion group, cells were infected with $B$. fragilis for the first hour and C. difficile added for the second hour; Substitution group, $C$. difficile were added for the first hour and B. fragilis added for the second hour. The cells were incubated at $37^{\circ} \mathrm{C}$ under anaerobic conditions for $2 \mathrm{~h}$ in total. (B) Transepithelial electrical resistance (TEER) values for Caco-2 cells in Control, B. fragilis ZY-312, C. difficile, Exclusion, Competition and Substitution groups. Control group, $2 \times 10^{4}$ Caco-2 cells were cultured without treatment. ZY-312 group, cells were incubated with $3 \times 10^{7} \mathrm{CFU} \mathrm{B.} \mathrm{fragilis} \mathrm{ZY-312.} \mathrm{C.} \mathrm{difficile}$ group, cells were incubated with $3 \times 10^{6} \mathrm{CFU}$ C. difficile. Competition group, cells were co-infected with $3 \times 10^{7} \mathrm{CFU} B$. fragilis and $3 \times 10^{6} \mathrm{CFU}$ C. difficile. Exclusion group, $B$. fragilis infected cells for the first hour and $C$. difficile for the second hour; Substitution group, $C$. difficile infected cells for the first hour and $B$. fragilis for the second hour. Data are shown as means \pm SEM. ${ }^{* * *} p<0.001$ by ANOVA.

relative abundance and OTU number, and rebalancing the composition (Figure 3 and Supplementary Figures S4, S5). A NMDS analysis (Figure 3A) demonstrated that our samples were qualified and acceptable, because the distributions of the inter-group samples were scattered much more discretely than those of the intra-group samples. Notably, the distribution of the microbiome in the MTZ group was obviously different from that in the BFT groups, indicating that the mechanism of MTZ treatment was different from B. fragilis. Similarly, the relative abundance of Verrucomicrobia (Figure 3B) and of its representative species Akkermansia muciniphila (Supplementary Figure S5B) in the cecum of antibiotic-treated mice (with or without spore challenge) was significantly increased after B. fragilis prophylactic treatment of CDI model mice.

\section{B. fragilis ZY-312 Inhibits C. difficile Adherence to HT-29 Cells}

In the present study, we examined whether $B$. fragilis could influence the adherence of $C$. difficile to colon cells, because colonization is a vital step for initiation of CDI pathogenesis in vivo. We found that $B$. fragilis significantly reduced the adherence of $C$. difficile to HT-29 cells (Figure 4A). All cells in the groups incubated with $B$. fragilis exhibited obvious resistance to $C$. difficile adherence, demonstrating that $B$. fragilis is capable of inhibiting $C$. difficile adherence to colon cells in vitro.

\section{B. fragilis ZY-312 Attenuates C. difficile-Induced Reduction in TEER in Caco-2 Cells}

As shown in Figure 4B, C. difficile induced a dramatic reduction in TEER of colon cell monolayers soon after infection, indicating that it is sufficiently toxic to cause gut barrier damage and increase gut permeability in vitro. A significant difference in the TEER value was observed at $4 \mathrm{~h}$ between the C. difficiletreated groups and the other groups $(p<0.001)$. Among the groups, the exclusion group presented the strongest protection, suggesting that colon cells pretreated with $B$. fragilis exhibit the best improvement of gut barrier permeability.

\section{B. fragilis ZY-312 Attenuates the Loss of Tight Junction Proteins and Mucins in Colon Cells Induced by C. difficile}

In the present study, we performed immunofluorescence staining to visualize ZO-1 and Muc-2 proteins and determine the protective effects of $B$. fragilis on tight junctions and barrier function in the epithelial system (Figure 5). The data showed strong green fluorescence in the control group and $B$. fragilistreated group, indicating that $\mathrm{ZO}-1$ protein was present in the intercellular spaces. In contrast, fluorescence of ZO-1 protein was almost absent in cells treated with $C$. difficile, demonstrating that the $C$. difficile-induced damage to the gut barrier probably involves tight junction disruption. Although $B$. fragilis did not provide obvious protection against loss of $\mathrm{ZO}-1$ protein in the competition group and substitution group, it did offer partial protection in the exclusion group.

Similarly, C. difficile significantly compromised MUC-2 expression and the reduction was attenuated by pretreatment with B. fragilis (Figure 5). The consistent results for PAS staining (Supplementary Figure S3) in HT-29 cells further confirmed the protective effects of $B$. fragilis on MUC-2 expression in the in vitro CDI model.

\section{B. fragilis ZY-312 Inhibits Colon Cell Apoptosis Induced by C. difficile}

According to the flow cytometry analysis (Figure 6), C. difficile induced significant early apoptosis of HT-29 cells (lower right 

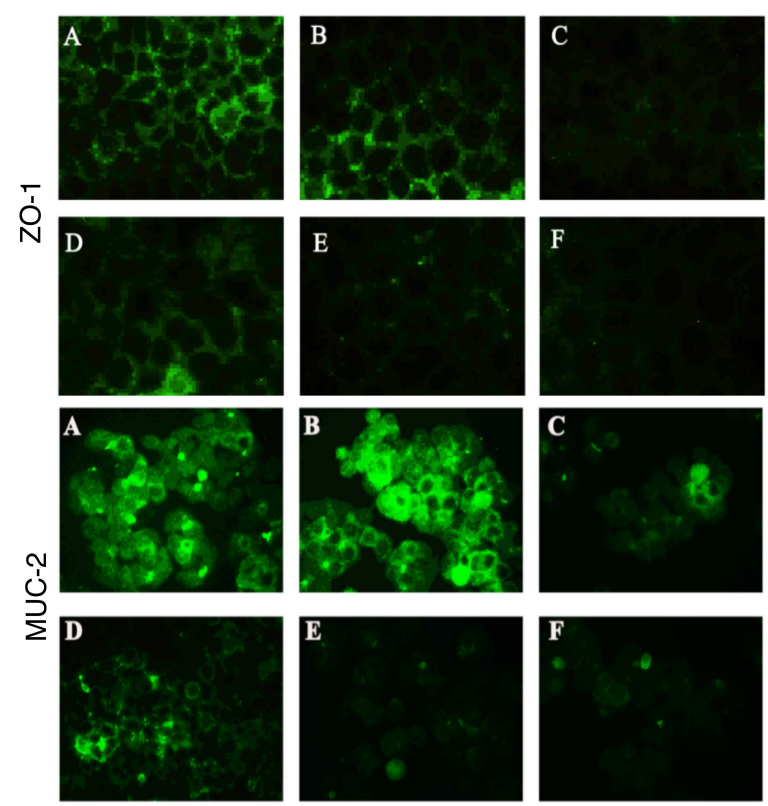

FIGURE 5 | Bacteroides fragilis ZY-312 increases expression of the tight junction protein ZO-1 and mucin MUC-2 in colon cells infected with $C$. difficile. Representative images for immunofluorescence staining of tight junction protein ZO-1 (top) in Caco-2 cells and mucin Muc-2 (middle) in HT-29 cells are shown for all groups. (A) Blank control group, $4 \times 10^{4}$ Caco-2 or HT-29 cells were cultured without treatment. (B) $B$. fragilis group, cells were incubated with $4 \times 10^{7}$ cfu $B$. fragilis. (C) C. difficile group, cells were incubated with $4 \times 10^{6}$ cfu C. difficile. (D) Exclusion group, cells were infected with $4 \times 10^{7}$ cfu $B$. fragilis for the first hour and $4 \times 10^{6}$ cfu $C$. difficile for the second hour. (E) Competition group, cells were co-infected with $B$. fragilis and C. difficile. (F) Substitution group, C. difficile was added for the first hour and $B$. fragilis added at the second hour. The cells were incubated at $37^{\circ} \mathrm{C}$ under anaerobic conditions for $2 \mathrm{~h}$.

quadrant), which was partly inhibited by B. fragilis. Specifically, pretreatment, co-incubation, and post-treatment with $B$. fragilis were all able to reduce the early apoptosis of HT-29 cells, demonstrating that $B$. fragilis improved cell survival in the in vitro CDI model. Microscopic observation of the IEC (Figure 6) and Vero (Supplementary Figure S3) cell morphology and viability provided consistent results. Treatment with $B$. fragilis significantly attenuated the damage, especially when the cells were pretreated.

\section{DISCUSSION}

Gut microbiota have received extensive attention in recent years, as their relationship with human health is far closer than previously expected. Clostridium difficile (C. difficile) is currently the single leading cause of inpatient infections and has become a public health threat due to its high morbidity and mortality rates (Kelly and LaMont, 2008). Antibiotic overuse, which disrupts the normal healthy gut microbiota of the host, is a huge risk factor for C. difficile infection (CDI) in the clinical setting. FMT is regarded as a potential bacteriotherapy for refractory CDI patients, especially for patients with recurrent disease (van Nood et al., 2013). A study reported that 13 of 16 patients recovered from CDI after performing duodenal infusion of donor feces just once. The bacterial diversity of the recovered patients increased and their microbiota composition became similar to the donors', with an increase in the relative abundance in Bacteroidetes species and a relative decrease in the abundance in Proteobacteria species (van Nood et al., 2013). However, there are several factors regarding FMT remaining to be settled, including the standards for a healthy donor, the storage of feces, and the risk of infusing opportunistic pathogens (Kelly and Tebas, 2018). To address these issues, Lawley et al tried to create a simpler and more targeted bacteriotherapy for CDI. They produced a simple mixture of six phylogenetically diverse intestinal bacteria which was able to clear the supershedding state of C. difficile 027/BI infected mice (Lawley et al., 2012), through helping the host re-establish a health-associated microbiota. A novel species of Bacteroides was included in that mixture, although it did not work in isolation nor mixed with Lactobacillus.

In this study, a single strain of $B$. fragilis was not able to rebuild a total health-associated microbiota net in CDI mice (Figure 3 and Supplementary Figure S4), although we did observe a significant increase in survival rate of the CDI mice prophylactically treated with $B$. fragilis. As a member of the commensal microbiota, $B$. fragilis plays an important role in microbe-host homeostasis, including regulation of immune system maturation and maintenance of immune tolerance (Round and Mazmanian, 2010). Moreover, B. fragilis protects mice from experimental colitis (Mazmanian et al., 2008) via a commensal symbiosis factor expressed in the outer membrane vesicles (Shen et al., 2012). This symbiosis factor was PSA, a zwitterionic polysaccharide, which protects the inflamed epithelium through boosting interleukin (IL)-10producing $\mathrm{CD}^{+}{ }^{+} \mathrm{T}$ cells and suppressing proinflammatory IL17-producing $\mathrm{CD}^{+}{ }^{+} \mathrm{T}$ cells (Mazmanian et al., 2008). Expansion and activation of Treg cells is important for maintain homeostasis after epithelial damage (Geuking et al., 2011), but PSA is not the only symbiosis factor produced by $B$. fragilis which influences epithelial homeostasis. Another study (Lee et al., 2013) revealed that $B$. fragilis has evolved to encode a special but highly conserved polysaccharide utilization locus for stable gut colonization. This genetic locus was named the commensal colonization factor (CCF), which not only helps B. fragilis penetrate the mucus and reside deep in colon crypt, but also creates a reservoir for resilience after enteric infections or antibiotic exposure. Previously we tracked the inhibition from B. fragilis to Vibrio parahaemolyticus infection in mice (Li et al., 2017), indicating $B$. fragilis also performed as the first-line defender to resist enteric pathogens. Here we observed obvious inhibition of $C$. difficile adherence by $B$. fragilis in vitro (Figure 4), and speculated that this might be an important preventive mechanism in vivo as well.

Impressively, another report (Hsiao et al., 2013) demonstrated that $B$. fragilis was able to ameliorate autism-related gastrointestinal and behavioral abnormalities in an autism mouse model, through restoration of gut barrier integrity and correction of abnormal microbiome composition. Our recent report (Zhang et al., 2018) further discovered that B. fragilis 

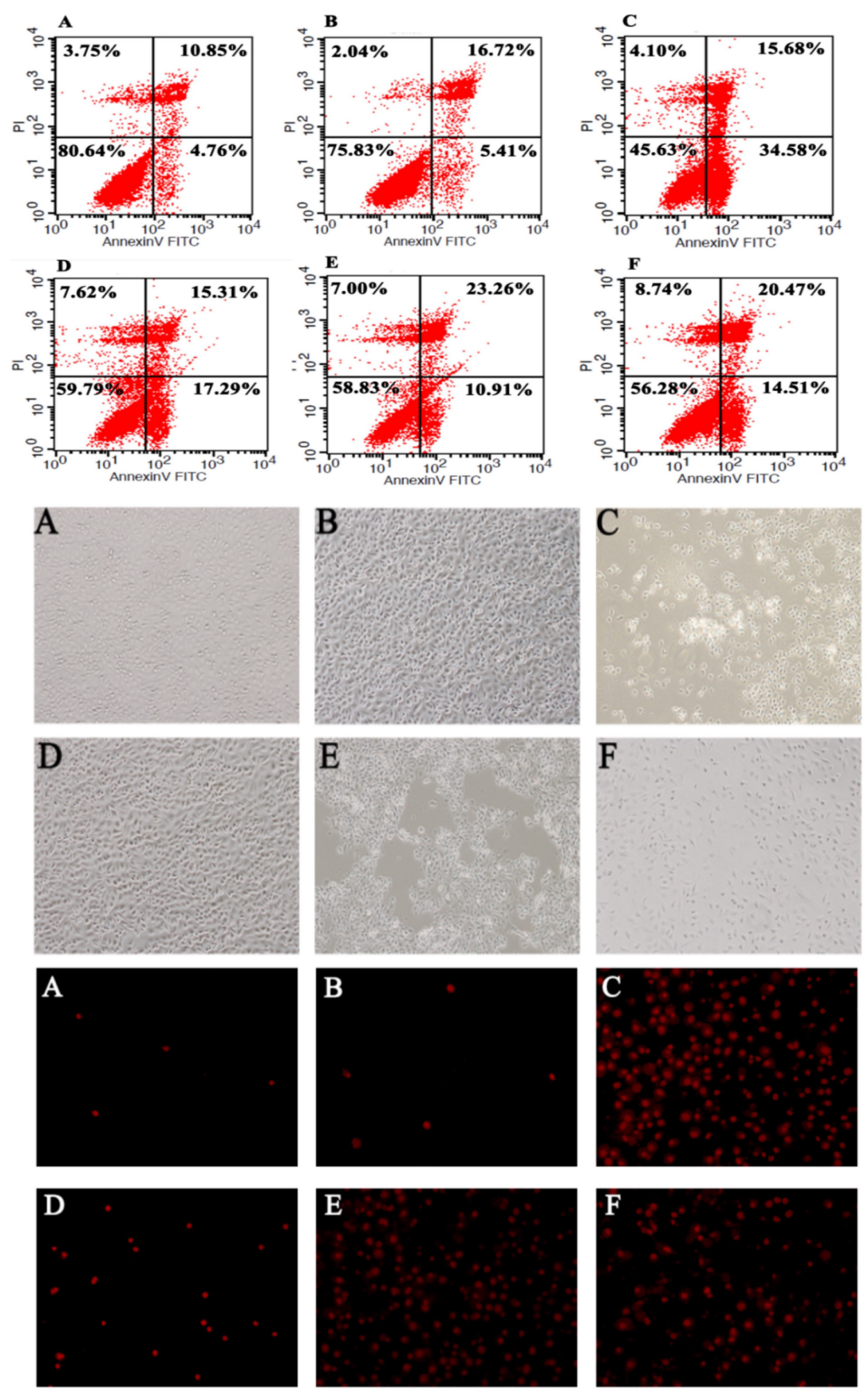

FIGURE 6 | Bacteroides fragilis ZY-312 inhibits colon cell apoptosis induced by C. difficile. Flow cytometry analyses (top) of HT-29 cells in all groups are shown. The lower right quadrant represents colon cells in early apoptosis. Microscopic observations (middle) of IEC-6 cell morphology and viability and PI staining (bottom) of IEC-6 cells in all groups are shown. (A) Blank control group, $5 \times 10^{5} \mathrm{HT}-29$ or IEC-6 cells were cultured without treatment. (B) $B$. fragilis group, cells were incubated with $5 \times 10^{8} \mathrm{cfu} B$. fragilis. (C) C. difficile group, cells were incubated with $5 \times 10^{7} \mathrm{cfu}$ C. difficile. (D) Exclusion group, cells were infected with $5 \times 10^{8} \mathrm{cfu} B$. fragilis for the first hour and $5 \times 10^{7}$ cfu C. difficile for the second hour. (E) Competition group, cells were co-infected with B. fragilis and C. difficile. (F) Substitution group, cells were infected with $C$. difficile for the first hour and $B$. fragilis for the second hour. The cells were incubated at $37^{\circ} \mathrm{C}$ under anaerobic conditions for 2 h. 
significantly ameliorated AAD-related diarrhea symptoms by increasing the abundance of specific commensal microbiota and restoring intestinal barrier function. As we know, $C$. difficile toxins, including Toxin A and Toxin B, mainly target intestinal epithelium through disrupting the cell cytoskeleton and inducing necrosis (Pothoulakis, 2000), thereby damaging colonic epithelial integrity. Therefore, we speculated that $B$. fragilis prevention of CDAD probably involved the improvement of gut barrier integrity. The results of Muc-2 and ZO-1 expression in in vivo (Figure 2) and in vitro (Figure 5) experiments suggested that $B$. fragilis was able to restore expression of mucins and tight junction proteins in epithelial cells during CDI.

Microbiome regulation was considered another possible mechanism by which $B$. fragilis prevents CDI. B. fragilis obviously increased intestinal bacterial diversity and commensal bacteria relative abundance in CDI mice (Figure 3 and Supplementary Figures S4, S5). A specific phenomenon was observed whereby the relative abundance of the Verrucomicrobia phylum was increased after $B$. fragilis prophylactic treatment group (Supplementary Figure S5). Comprising 98\% of the Verrucomicrobia phylum, A. muciniphila is a Gram-negative, mucin-utilizing commensal bacterium that resides in the mucus layer of the human intestinal tract. It has been reported that A. muciniphila has evolved to specialize in degrading and utilizing mucus to provide a source of carbon and nitrogen. Belzer et al. (2017) co-cultured A. muciniphila with the non-mucusdegrading butyrate-producing bacteria Eubacterium hallii and found syntrophic growth and butyrate production. This study reminds us of the probability that there might also exist bacterial cross-feeding interactions between A. muciniphila and $B$. fragilis, since $B$. fragilis is the major source of shortchain fatty acids in the intestinal tract (Wang et al., 2017). Briefly, cross-feeding is one microorganism utilizing the end products or carbohydrate breakdown products offered by another microorganism (Rios-Covian et al., 2016), which is common among gut microbiota. The exact mechanisms of the interactions between $B$. fragilis, A. muciniphila, and the intestinal epithelium remain to be clearly established, but the maintenance and growth of A. muciniphila after B. fragilis pretreatment is believed to take part in restoring gut barrier integrity during $C$. difficile pathogenesis.

With the initial identification of an inverse relationship between B. fragilis and C. difficile (Goldberg et al., 2014), and based on the theory of gut barrier disruption by CDI and protection by $B$. fragilis, we have proven that $B$. fragilis prophylactic treatment can ameliorate morbidity and mortality in a CDI mouse model. This is likely through inhibiting $C$. difficile colonization, modulating gut microbiota and alleviating barrier

\section{REFERENCES}

Belzer, C., Chia, L. W., Aalvink, S., Chamlagain, B., Piironen, V., Knol, J., et al. (2017). Microbial metabolic networks at the mucus layer lead to diet-independent butyrate and vitamin b12 production by intestinal symbionts. mBio 8:e00770-17. doi: 10.1128/mBio. 00770-17

Bron, P. A., Kleerebezem, M., Brummer, R. J., Cani, P. D., Mercenier, A., MacDonald, T. T., et al. (2017). Can probiotics modulate human disease by destruction, thus relieving epithelial stress and pathogenic colitis. The present findings demonstrated the direct protection against CDI provided by a single strain of commensal bacteria, which lay the foundations for exploring the optimal bacteriotherapy for CDI prophylaxis and treatment. They also provide deep insight into microbe-microbe and microbeepithelium interactions. However, further studies are needed to clarify the molecular mechanisms involved in the protective roles of $B$. fragilis in gut barrier maintenance and microbe-microbe interactions.

\section{AUTHOR CONTRIBUTIONS}

HD performed the experiments with bacteria and mice, analyzed the data, and wrote the manuscript. SY performed the experiments with bacteria and mice and analyzed the data. KQ and $\mathrm{ZZ}$ performed the experiments with mice. YZ performed the experiments with bacteria and cells, analyzed the data, and contributed to revising the manuscript. YL and YW provided the bacteria and contributed to revising the manuscript. YB contributed to revising the manuscript. HF and $\mathrm{XZ}$ designed the experiments, analyzed the data, and contributed to revising the manuscript. FZ provided overall directions and contributed to revising the manuscript.

\section{FUNDING}

This work was supported by Special fund of Science and Technology development of Guangdong Province (No. 2016B090918064), Special fund of Public Welfare research and capacity building of Guangdong Province (No. 2016A020217010), Special Project on the integration of Industry, Education and Research, collaborated with innovation of Guangzhou (No. 201605122107130), Pear River Nova Program on the innovation of environmental construction of Guangzhou (No. 201806010010), and the National Natural Science Foundation of China (Nos. 31872630 and 81402037), and Innovation Leader Team Program of Guangzhou (No. 201809010014).

\section{SUPPLEMENTARY MATERIAL}

The Supplementary Material for this article can be found online at: https://www.frontiersin.org/articles/10.3389/fmicb. 2018.02976/full\#supplementary-material

impacting intestinal barrier function? Br. J. Nutr. 117, 93-107. doi: 10.1017/ S0007114516004037

Buonomo, E. L., Cowardin, C. A., Wilson, M. G., Saleh, M. M., Pramoonjago, P., and Petri, W. A. Jr. (2016). Microbiota-regulated IL-25 increases eosinophil number to provide protection during clostridium difficile infection. Cell Rep. 16, 432-443. doi: 10.1016/j.celrep.2016.06.007

Crobach, M. J. T., Vernon, J. J., Loo, V. G., Kong, L. Y., Pechine, S., Wilcox, M. H., et al. (2018). Understanding clostridium difficile colonization. Clin. Microbiol. Rev. 31:e00021-17. doi: 10.1128/CMR.00021-17 
Deng, H., Li, Z., Tan, Y., Guo, Z., Liu, Y., Wang, Y., et al. (2016). A novel strain of Bacteroides fragilis enhances phagocytosis and polarises M1 macrophages. Sci. Rep. 6:29401. doi: 10.1038/srep29401

Geuking, M. B., Cahenzli, J., Lawson, M. A., Ng, D. C., Slack, E., Hapfelmeier, S., et al. (2011). Intestinal bacterial colonization induces mutualistic regulatory $\mathrm{T}$ cell responses. Immunity 34, 794-806. doi: 10.1016/j.immuni.2011.03.021

Goldberg, E., Amir, I., Zafran, M., Gophna, U., Samra, Z., Pitlik, S., et al. (2014). The correlation between Clostridium-difficile infection and human gut concentrations of Bacteroidetes phylum and clostridial species. Eur. J. Clin. Microbiol. Infect. Dis. 33, 377-383. doi: 10.1007/s10096-013-1966-x

He, M., Miyajima, F., Roberts, P., Ellison, L., Pickard, D. J., Martin, M. J., et al. (2013). Emergence and global spread of epidemic healthcare-associated Clostridium difficile. Nat. Genet. 45, 109-113. doi: 10.1038/ng.2478

Hsiao, E. Y., McBride, S. W., Hsien, S., Sharon, G., Hyde, E. R., McCue, T., et al. (2013). Microbiota modulate behavioral and physiological abnormalities associated with neurodevelopmental disorders. Cell 155, 1451-1463. doi: 10 . 1016/j.cell.2013.11.024

Hutton, M. L., Mackin, K. E., Chakravorty, A., and Lyras, D. (2014). Small animal models for the study of Clostridium difficile disease pathogenesis. FEMS Microbiol. Lett. 352, 140-149. doi: 10.1111/1574-6968.12367

Kelly, B. J., and Tebas, P. (2018). Clinical practice and infrastructure review of fecal microbiota transplantation for clostridium difficile infection. Chest 153, 266-277. doi: 10.1016/j.chest.2017.09.002

Kelly, C. P., and LaMont, J. T. (2008). Clostridium difficile-more difficult than ever. N. Engl. J. Med. 359, 1932-1940. doi: 10.1056/NEJMra0707500

Kuczynski, J., Stombaugh, J., Walters, W. A., Gonzalez, A., Caporaso, J. G., and Knight, R. (2012). Using QIIME to analyze 16S rRNA gene sequences from microbial communities. Curr. Protoc. Microbiol. 36, 10.7.1-10.7.20. doi: 10. 1002/9780471729259.mc01e05s27

Lawley, T. D., Clare, S., Walker, A. W., Stares, M. D., Connor, T. R., Raisen, C., et al. (2012). Targeted restoration of the intestinal microbiota with a simple, defined bacteriotherapy resolves relapsing Clostridium difficile disease in mice. PLoS Pathog. 8:e1002995. doi: 10.1371/journal.ppat.1002995

Lee, S. M., Donaldson, G. P., Mikulski, Z., Boyajian, S., Ley, K., and Mazmanian, S. K. (2013). Bacterial colonization factors control specificity and stability of the gut microbiota. Nature 501, 426-429. doi: 10.1038/nature12447

Lessa, F. C., Mu, Y., Bamberg, W. M., Beldavs, Z. G., Dumyati, G. K., Dunn, J. R., et al. (2015). Burden of Clostridium difficile infection in the United States. N. Engl. J. Med. 372, 825-834. doi: 10.1056/NEJMoa1408913

Li, Z., Deng, H., Zhou, Y., Tan, Y., Wang, X., Han, Y., et al. (2017). Bioluminescence imaging to track Bacteroides fragilis inhibition of vibrio parahaemolyticus infection in mice. Front. Cell. Infect. Microbiol. 7:170. doi: 10.3389/fcimb.2017. 00170

Mazmanian, S. K., Round, J. L., and Kasper, D. L. (2008). A microbial symbiosis factor prevents intestinal inflammatory disease. Nature 453, 620-625. doi: 10 . 1038/nature07008

Mills, J. P., Rao, K., and Young, V. B. (2018). Probiotics for prevention of Clostridium difficile infection. Curr. Opin. Gastroenterol. 34, 3-10. doi: 10.1097/ MOG.0000000000000410

Mushtaq, A. (2018). New clinical recommendations for Clostridium difficile. Lancet Infect. Dis. 18:384. doi: 10.1016/S1473-3099(18)30180-4

Pothoulakis, C. (2000). Effects of Clostridium difficile toxins on epithelial cell barrier. Ann. N. Y. Acad. Sci. 915, 347-356. doi: 10.1111/j.1749-6632.2000. tb05263.x

Rineh, A., Kelso, M. J., Vatansever, F., Tegos, G. P., and Hamblin, M. R. (2014). Clostridium difficile infection: molecular pathogenesis and novel therapeutics. Expert Rev. Anti Infect. Ther. 12, 131-150. doi: 10.1586/14787210.2014.866515
Rios-Covian, D., Ruas-Madiedo, P., Margolles, A., Gueimonde, M., de Los ReyesGavilan, C. G., and Salazar, N. (2016). Intestinal short chain fatty acids and their link with diet and human health. Front. Microbiol. 7:185. doi: 10.3389/fmicb. 2016.00185

Round, J. L., and Mazmanian, S. K. (2010). Inducible Foxp3+ regulatory T-cell development by a commensal bacterium of the intestinal microbiota. Proc. Natl. Acad. Sci. U.S.A. 107, 12204-12209. doi: 10.1073/pnas.090912 2107

Shen, N. T., Maw, A., Tmanova, L. L., Pino, A., Ancy, K., Crawford, C. V., et al. (2017). Timely use of probiotics in hospitalized adults prevents Clostridium difficile infection: a systematic review with meta-regression analysis. Gastroenterology 152, 1889.e9-1900.e9. doi: 10.1053/j.gastro.2017. 02.003

Shen, Y., Giardino Torchia, M. L., Lawson, G. W., Karp, C. L., Ashwell, J. D., and Mazmanian, S. K. (2012). Outer membrane vesicles of a human commensal mediate immune regulation and disease protection. Cell Host Microbe 12, 509-520. doi: 10.1016/j.chom.2012.08.004

Sorg, J. A., and Sonenshein, A. L. (2008). Bile salts and glycine as cogerminants for Clostridium difficile spores. J. Bacteriol. 190, 2505-2512. doi: 10.1128/JB. 01765-07

Tang, C., Cui, L., Xu, Y., Xie, L., Sun, P., Liu, C., et al. (2016). The incidence and drug resistance of Clostridium difficile infection in Mainland China: a systematic review and meta-analysis. Sci. Rep. 6:37865. doi: 10.1038/srep37865

van Nood, E., Vrieze, A., Nieuwdorp, M., Fuentes, S., Zoetendal, E. G., de Vos, W. M., et al. (2013). Duodenal infusion of donor feces for recurrent Clostridium difficile. N. Engl. J. Med. 368, 407-415. doi: 10.1056/NEJMoa120 5037

Wang, Y., Deng, H., Li, Z., Tan, Y., Han, Y., Wang, X., et al. (2017). Safety evaluation of a novel strain of Bacteroides fragilis. Front. Microbiol. 8:435. doi: 10.3389/fmicb.2017.00435

Winston, J. A., and Theriot, C. M. (2016). Impact of microbial derived secondary bile acids on colonization resistance against Clostridium difficile in the gastrointestinal tract. Anaerobe 41, 44-50. doi: 10.1016/j.anaerobe.2016.05.003

Yan, W., Shin, K. S., Wang, S. J., Xiang, H., Divers, T., McDonough, S., et al. (2014). Equine hyperimmune serum protects mice against Clostridium difficile spore challenge. J. Vet. Sci. 15, 249-258. doi: 10.4142/jvs.2014.15. 2.249

Zhang, W., Zhu, B., Xu, J., Liu, Y., Qiu, E., Li, Z., et al. (2018). Bacteroides fragilis protects against antibiotic-associated diarrhea in rats by modulating intestinal defenses. Front. Immunol. 9:1040. doi: 10.3389/fimmu.2018.01040

Conflict of Interest Statement: B. fragilis ZY-312 belongs to Guangzhou ZhiYi Biotechnology Co., Ltd. Any use of B. fragilis ZY-312 without the permission of Guangzhou ZhiYi Biotechnology Co., Ltd. is illegal. YL and YW were employed by Guangzhou ZhiYi Biotechnology Co., Ltd.

The remaining authors declare that the research was conducted in the absence of any commercial or financial relationships that could be construed as a potential conflict of interest.

Copyright (c) 2018 Deng, Yang, Zhang, Qian, Zhang, Liu, Wang, Bai, Fan, Zhao and Zhi. This is an open-access article distributed under the terms of the Creative Commons Attribution License (CC BY). The use, distribution or reproduction in other forums is permitted, provided the original author(s) and the copyright owner(s) are credited and that the original publication in this journal is cited, in accordance with accepted academic practice. No use, distribution or reproduction is permitted which does not comply with these terms. 